\title{
Proteasome inhibitor bortezomib (PS-341) enhances RANKL- induced MDA-MB-231 breast cancer cell migration
}

\author{
LINGYUN ZHANG, YUEE TENG, YE ZHANG, JING LIU, LING XU, \\ JINGLEI QU, KEZUO HOU, YUNPENG LIU* and XIUJUAN QU*
}

Department of Medical Oncology, The First Hospital of China Medical University, Shenyang 110001, P.R. China

Received September 16, 2011; Accepted November 11, 2011

DOI: $10.3892 / \mathrm{mmr} .2011 .678$

\begin{abstract}
The receptor activator of nuclear factor $\kappa \mathrm{B}$ ligand/ receptor activator of nuclear factor $\kappa B$ (RANKL/RANK) pathway is crucial for the migration of RANK-expressing cancer cells. The ubiquitin-proteasome protein degradation pathway plays a significant role in tumor metastasis. However, the relationship between these two pathways in tumor cell migration is unclear. In the present study, we explored the effect of the proteasome inhibitor bortezomib (PS-341) on RANKL-induced MDA-MB-231 breast cancer cell migration. Transwell migration assay showed that RANKL-induced MDA-MB-231 cell migration was significantly blocked by the decoy receptor osteoprotegerin (OPG), and was also inhibited by the PI3-K inhibitor LY294002. Western blotting results showed that Akt was rapidly activated by soluble RANKL treatment. PS-341 significantly enhanced RANKL-induced MDA-MB-231 cell migration. Further study showed that the enhancement of migration by PS-341 involved upregulation of activated Akt and RANK. Our results for the first time support the theory that PS-341 treatment may be unsuitable for RANK-positive breast cancer patients.
\end{abstract}

\section{Introduction}

Breast cancer is the most common malignancy in women. Although early-stage breast cancers are not life threatening, development of metastatic breast cancer is responsible for the majority of cancer-related deaths and the poor quality of life of patients. Currently available treatments are unable to eradicate metastatic cancer, and the survival rate for these patients is

Correspondence to: Dr Yunpeng Liu and Dr Xiujuan Qu, Department of Medical Oncology, The First Hospital of China Medical University, No. 155 North Nanjing Street, Heping, Shenyang 110001, P.R. China

E-mail: cmuliuyunpeng@yahoo.cn

E-mail: xiujuanqu@yahoo.com

${ }^{*}$ Contributed equally

Key words: bortezomib, receptor activator for nuclear factor $\kappa \mathrm{B}$ ligand, migration, breast cancer low. Further therapeutic advances require new strategies based on a fundamental understanding of breast cancer biology.

Recent studies have shown that the receptor activator for nuclear factor $\kappa \mathrm{B}$ ligand/receptor activator for nuclear factor $\kappa \mathrm{B}$ (RANKL/RANK) pathway is critical for RANK-expressing cancer cells to home to bones, and RANKL increased migration of RANK-expressing cancer cells, including breast cancer cells (1-3). However, the regulatory mechanism of the RANKL/RANK pathway in cancer cell migration remains largely unknown.

The ubiquitin-proteasome protein degradation pathway plays an essential role in the orderly proteolysis of intracellular proteins. In cancer cells, this pathway affects numerous activities that are important for tumor growth and metastasis (4). The proteasome inhibitor bortezomib (PS-341) has been licensed for the treatment of refractory multiple myeloma and mantle cell lymphoma. Although PS-341 is active against breast cancer cell lines $(5,6)$, the effect of PS-341 on RANKLinduced breast cancer cell migration has yet to be clarified.

In the present study, we explored the effect of PS-341 on the RANKL/RANK pathway and demonstrated for the first time that PS-341 enhanced RANKL-mediated MDA-MB-231 cell migration. This increased migration was associated with upregulation of RANK expression and phospho-Akt levels.

\section{Materials and methods}

Cell culture. The MDA-MB-231 breast cancer cell line was obtained from the Type Culture Collection of the Chinese Academy of Sciences (Shanghai, China). MDA-MB-231 cells were grown in Leibovitz L-15 medium (Gibco, Carlsbad, CA, USA) containing $10 \% \mathrm{FBS}$, according to the recommendations of the supplier.

Reagents and antibodies. Recombinant soluble RANKL (sRANKL) and recombinant human osteoprotegerin (OPG; rOPG) were purchased from Cytolab/Peprotech Asia (USA) and PS-341 was purchased from Millennium Pharmaceuticals, Inc. (Cambridge, MA, USA). The specific PI3-K inhibitor LY294002 was obtained from Sigma (St. Louis, MO, USA). Rabbit anti-actin antibodies were obtained from Santa Cruz Biotechnology (Santa Cruz, CA, USA). Rabbit anti-Akt and rabbit anti-p-Akt (Ser473) were obtained from Cell Signaling Technology (Danvers, MA, USA). 
A

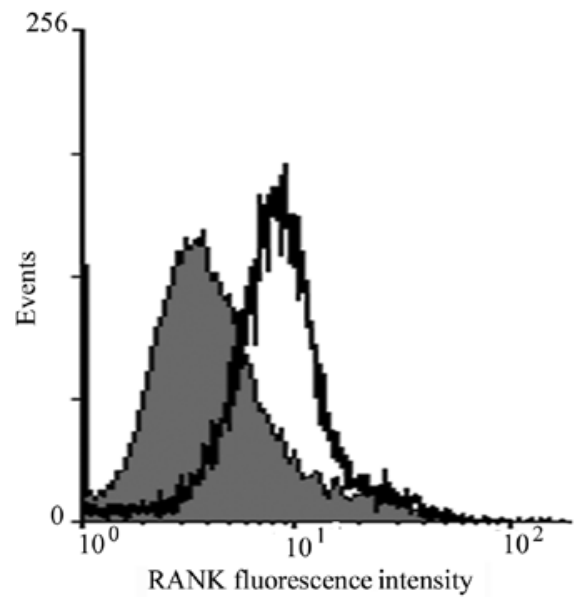

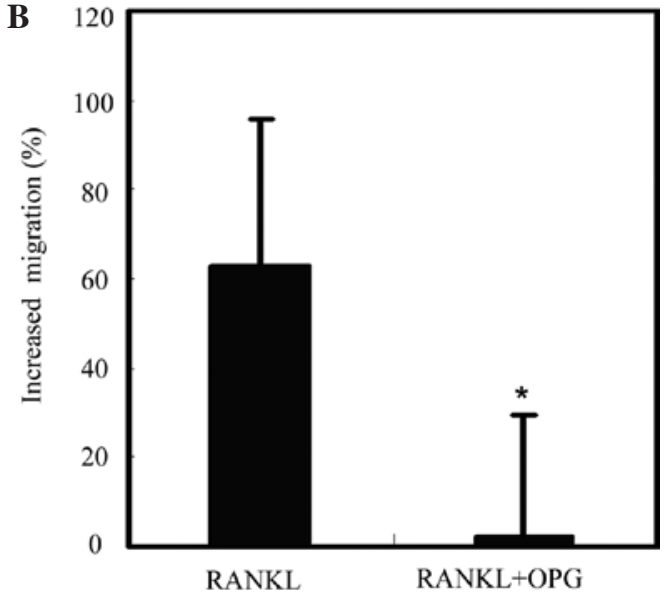

Figure 1. RANKL increases RANK-expressing MDA-MB-231 cell migraton. (A) RANK expression on the surface of MDA-MB-231 cells was examined by flow cytomety. Isotype control staining is shown in grey. (B) MDA-MB-231 cells were incubated with sRANKL (2 $\mu \mathrm{g} / \mathrm{ml}) \mathrm{with}$ or without rOPG (10 $\mu \mathrm{g} / \mathrm{ml})$, separately, and the in vitro migration activity was measured with the Transwell after $22 \mathrm{~h}$. Results are expressed as the means $\pm \mathrm{SD}$. The data are representative results from at least three independent experiments. ${ }^{*} \mathrm{P}<0.05$ compared to the control.

RANK expression analysis. The MDA-MB-231 cells were seeded at $5 \times 10^{5}$ cells/well in 6-well plates. Surface RANK expression was determined by flow cytometry following incubation with either $25 \mu \mathrm{g} / 10^{6}$ cells mouse anti-RANK antibody (R\&D Systems, Minneapolis, MN, USA) or isotype control (R\&D) followed by FITC-conjugated anti-mouse secondary antibody. Fluorescence was assessed using a FAScan sorter (BD Biosciences, San Jose, CA, USA). Finally, the data were analyzed with WinMDI software.

Western blotting. To prepare total cell lysates, cell pellets were washed twice with ice-cold PBS and solubilized in $1 \%$ Triton lysis buffer [1\% Triton X-100, $50 \mathrm{mM}$ Tris- $\mathrm{HCl}(\mathrm{pH} 7.4)$, $150 \mathrm{mM} \mathrm{NaCl}, 10 \mathrm{mM}$ EDTA, $100 \mathrm{mM} \mathrm{NaF}, 1 \mathrm{mM} \mathrm{Na} \mathrm{VO}_{4}$, $1 \mathrm{mM}$ phenylmethylsulfonyl fluoride and $2 \mu \mathrm{g} / \mathrm{ml}$ aprotinin] on ice. Cell lysates were centrifuged at 12,000 x $\mathrm{g}$ for $30 \mathrm{~min}$ at $4^{\circ} \mathrm{C}$ and the supernatants were separated. Proteins were eluted by heat treatment at $100^{\circ} \mathrm{C}$ for $5 \mathrm{~min}$ with $3 \mathrm{X}$ sampling buffer. Total proteins $(30-50 \mu \mathrm{g})$ were subjected to SDS-polyacrylamide gel electrophoresis (SDS-PAGE) and electronically transferred to nitrocellulose membranes. After blocking with $5 \%$ skim milk in TBST $[10 \mathrm{mM}$ Tris (pH 7.4), $150 \mathrm{mM} \mathrm{NaCl}$ and $0.1 \%$ Tween-20], the blots were probed with the indicated primary antibodies at $4^{\circ} \mathrm{C}$ overnight, followed by the horseradish peroxidase-conjugated specific goat antimouse or goat anti-rabbit secondary antibody as indicated for $30 \mathrm{~min}$ at room temperature. Finally, proteins were detected using an enhanced chemiluminescence reagent (SuperSignal Western Pico Chemiluminescent Substrate; Pierce, USA) and visualized with Electrophoresis Gel Imaging Analysis System (DNR Bio-Imaging Systems, Jerusalem, Israel).

Transwell migration assay. Cells were washed twice in culture medium with $0.1 \%$ bovine serum albumin. A 24-well chemotaxis chamber (8- $\mu \mathrm{m}$ pore size; Corning, NY, USA) was used for this experiment. Prior to performing the migration assay, cells were pre-treated with different concentrations of inhibitors or the appropriate solvent control (dimethyl sulfoxide; DMSO) for $60 \mathrm{~min}$. A volume of $200 \mu \mathrm{l}\left(1 \times 10^{5}\right.$ cells $\left./ \mathrm{ml}\right)$ from each sample was loaded onto the upper well. The medium $(0.5 \mathrm{ml})$ containing sRANKL with or without the OPG or DMSO was added to the bottom well. The plates were incubated for $16 \mathrm{~h}$ at $37^{\circ} \mathrm{C}$. Following incubation, the porous inserts were carefully removed and the cells on the lower surface of the membrane were stained and counted in at least five different fields. The results were expressed as the percentage of migrated cells as compared to the control (untreated cells). Each experiment was performed at least three times.

Statistical analysis. Experimental data are presented as the means \pm standard deviation. The significance of the difference between the groups was assessed by the Student's two-tailed $\mathrm{t}$-test, and $\mathrm{P}<0.05$ was considered significant in all statistical analyses. All mean values were calculated from at least three independent experiments. Statistical analyses were carried out using SPSS 16.0 for Windows (SPSS, Inc., Chicago, IL, USA).

\section{Results}

RANKL and RANK interaction direct the migration of $M D A-M B-231$ breast cancer cells. Firstly, flow cytometry was used to examine RANK expression in MDA-MB-231 cells. The result showed that MDA-MB-231 cells expressed RANK on the cell surface (Fig. 1A). Subsequently, stimulation of the MDA-MB-231 cells by sRANKL significantly increased cell migration. Moreover, treatment of cells with decoy receptor of RANKL, OPG, significantly blocked RANKL-induced cell migration (Fig. 1B).

Akt is involved in RANKL-induced MDA-MB-231 cell migration. Next, the downstream signaling of RANKL/RANK in MDA-MB-231 cells was examined. Since the PI3-K/Akt pathway is closely correlated with cell invasion and migration (2,7-10), the effect of Akt on the RANKL/RANK pathway was explored. The results showed that Akt was transiently activated by sRANKL in MDA-MB-231 cells (Fig. 2A). The sRANKL-induced migration was significantly inhibited by the PI3-K inhibitor LY294002 (50 $\mu \mathrm{M})$ (Fig. 2B). 
A

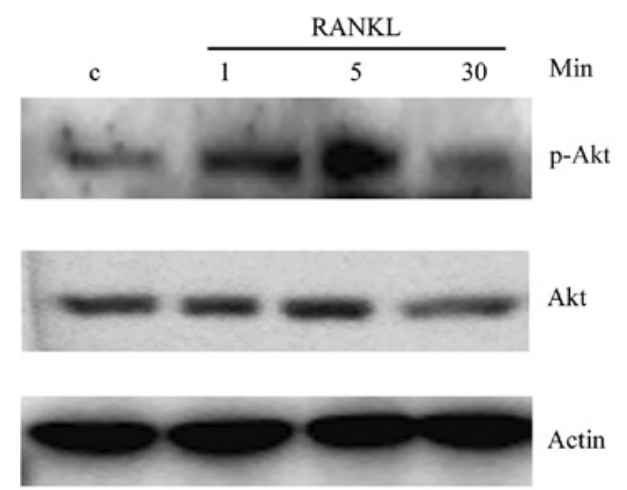

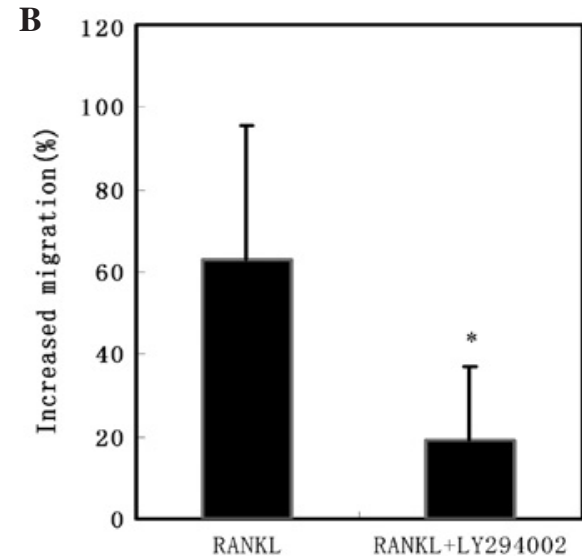

Figure 2. Akt is involved in RANKL-induced breast cancer cell migration. (A) MDA-MB-231 cells were incubated with sRANKL ( $2 \mu \mathrm{g} / \mathrm{ml})$ for indicated time intervals, while pAkt/Akt and actin were examined by Western blot analysis. (B) MDA-MB-231 cells were incubated with RANKL (2 $\mu \mathrm{g} / \mathrm{ml})$ with or without LY294002 (50 mM, PI3-K inhibitor), and the migration activity was measured with the Transwell after $22 \mathrm{~h}$. Results are expressed as the means \pm SD. The data are representative results from at least three independent experiments. ${ }^{*} \mathrm{P}<0.05$ compared to the control.

A

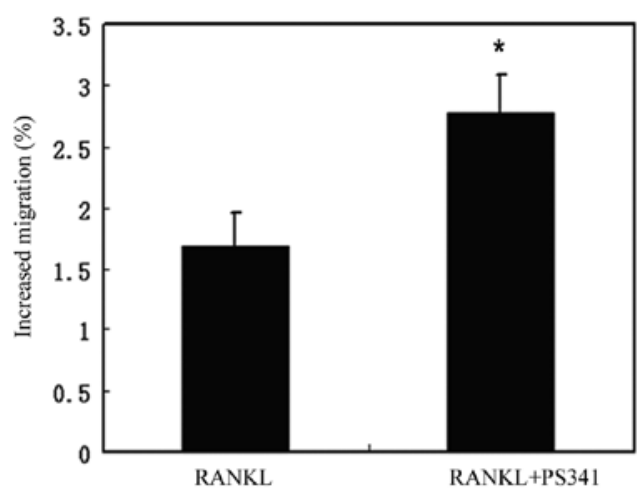

C

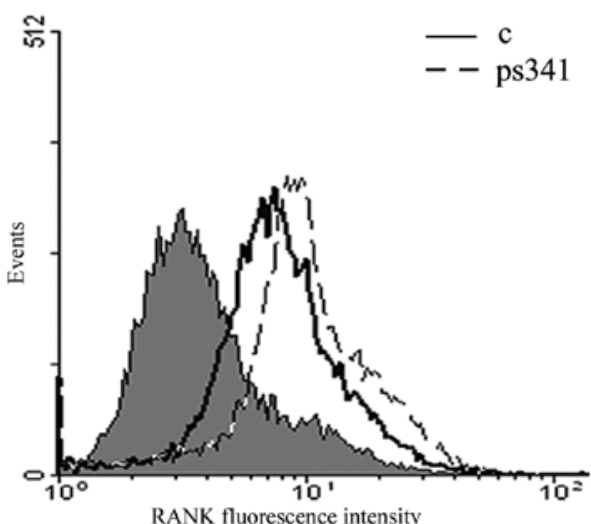

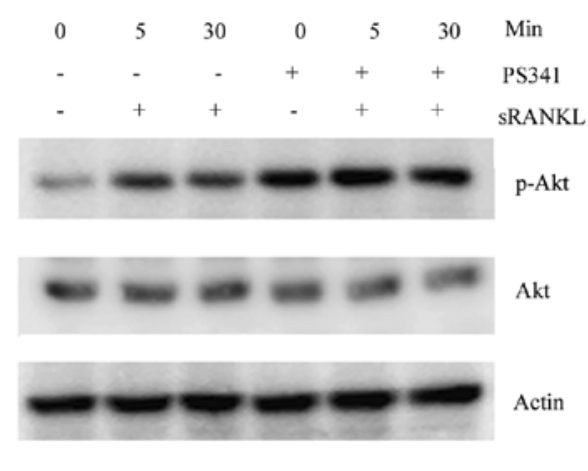

D

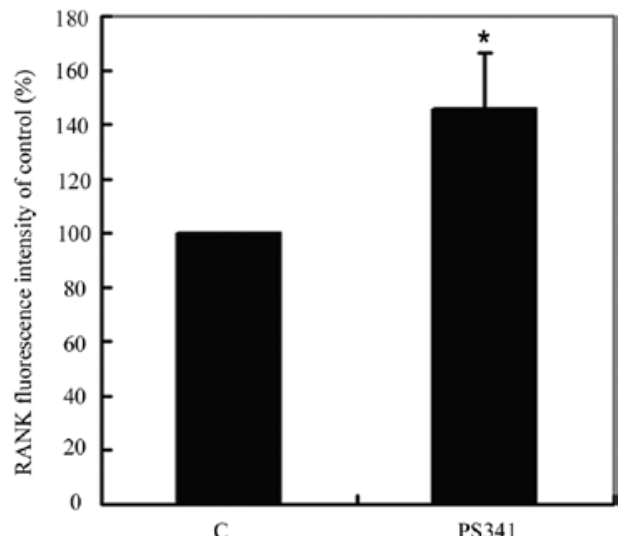

C

PS341

Figure 3. PS-341 enhances RANKL-induced MDA-MB-231 cell migration by upregulation of RANK and phospho-Akt. (A) MDA-MB-231 cells were incubated with sRANKL $(2 \mu \mathrm{g} / \mathrm{ml})$, with or without pre-treatment with PS-341 $(10 \mathrm{nM})$ for $4 \mathrm{~h}$, and the migration activity was measured with the Transwell after $22 \mathrm{~h}$. Results are expressed as the means $\pm \mathrm{SD}$. The data are representative results from at least three independent experiments. "P $<0.05$ compared to control (B) MDA-MB-231 cells were incubated with sRANKL $(2 \mu \mathrm{g} / \mathrm{ml})$ for indicated time intervals, with or without pre-treatment with PS-341 (10 $\mathrm{nM})$ for $4 \mathrm{~h}$, while pAkt/Akt and actin were examined by Western blot analysis. (C) MDA-MB-231 cells were treated with or without PS-341 (10 nM) for $24 \mathrm{~h}$, and RANK expression on the surface of MDA-MB-231 cells was detected by flow cytomety. The grey area indicates isotype control staining and the lines indicate treated experimental cells. (D) Quantitative representation of the cell surface expression of RANK. ${ }^{*} \mathrm{P}<0.05$.

These results indicated that the PI3-K/Akt pathway was involved in RANKL-induced MDA-MB-231 breast cancer cell migration.

PS-341 enhances RANKL-induced MDA-MB-231 cell migration by upregulation of RANK and phospho-Akt. Transwell migration assay was used to explore the effect of PS-341 on RANKL-induced MDA-MB-231 cell migration. The results showed that pre-treatment with PS-341 for $4 \mathrm{~h}$ markedly increased RANKL-induced MDA-MB-231 cell migration (Fig. 3A). Western blotting results demonstrated that pre-treatment with PS-341 for $4 \mathrm{~h}$, followed by exposure to sRANKL, 
significantly increased the p-Akt levels (Fig. 3B). FACS results showed that after treatment with PS-341 for $24 \mathrm{~h}$, RANK expression on MDA-MB-231 cells was upregulated (Fig. 3C and D).

These results indicated that PS-341 enhanced RANKLinduced MDA-MB-231 cell migration by upregulation of RANK and phospho-Akt levels.

\section{Discussion}

The RANKL/RANK pathway is considered the key pathway in the regulation of osteoclast and lymphocyte proliferation and survival. RANKL interacts with RANK and in turn recruits tumor necrosis factor receptor-associated factors (11) leading to activation of NF- $\mathrm{NB}$, c-Jun N-terminal kinase, p38, ERK and Akt (12-14). As recently demonstrated, RANK has been found to be expressed in several solid tumors, such as breast cancer, prostate cancer, malignant bone tumors, multiple myeloma and squamous cell carcinoma $(1,15)$, and RANKL directs RANK-expressing cancer cells to preferentially migrate into bone. However, the exact signaling pathways in tumor cell migration need to be elucidated. Tang et al (16) reported that Src and MAPK were involved in RANKL-induced MDA-MB231 cell migration. In T47D breast cancer cells, ERK and Akt were involved in RANKL-induced cell migration (1), while in C42b prostate cancer cells, ERK, but not Akt, was involved in the RANKL/RANK pathway (3). These data showed that RANKL/RANK signaling pathways vary in different tumor cell types. In the present study, we firstly showed that Akt was involved in RANKL-induced MDA-MB-231 cell migration.

Proteasome is involved in the targeted elimination of damaged and regulatory proteins, such as transcription factors, signaling molecules and cell cycle inhibitors (17). The inhibition of the proteasome results in the abnormal accumulation of many intracellular proteins, thereby disrupting cellular homeostasis. In osteoclast differentiation and survival, the RANKL/RANK pathway was found to be regulated by the proteasome pathway $(18,19)$. Tumor cells have been reported to be more sensitive to proteasome inhibition than normal cells, thus making proteasome a potential novel target for cancer treatment (20).

PS-341 is a selective and reversible proteasome inhibitor that results in stabilization of cell cycle regulatory proteins, inhibition of NF- $\mathrm{BB}$ activation, induction of apoptosis and override of Bcl-2 resistance and anti-angiogenesis (21). Moreover, PS-341 exhibits a wide range of antitumor activity and increases the activity of multiple chemotherapeutic agents $(5,6,22,23)$. Although PS-341 was found to be active against breast cancer cell lines, in vivo models and phase I clinical trials have found it ineffective for breast cancer when used as a single agent (24), and limited efficacy of PS-341 has been noted in combination with several chemotherapeutic agents $(25,26)$.

In the present study, the role of low-dose PS-341 (10 nM) in RANKL-induced MDA-MB-231 cell migration was explored. The results showed that PS-341 significantly enhanced RANKL-induced MDA-MB-231 cell migration. Furthermore, PS-341 treatment resulted in the upregulation of RANK expression and p-Akt levels, which suggests that the activation of the RANKL/RANK/Akt pathway may contribute to increased RANKL-induced breast cancer cell migration. Since a recent study showed that RANK expression may predict accelerated skeletal metastasis-free survival and poor prognosis in breast cancer patients (27), our results suggest that PS-341 treatment may be unsuitable for RANK-positive breast cancer patients.

Taken together, our results provide evidence that PS-341 increases the RANKL-induced MDA-MB-231 cell migration. Further study is required to confirm the effect of PS-341 in RANKL-expressing cancer cells.

\section{Acknowledgements}

The authors thank their clinical and laboratory colleagues who contributed to the research. This study was supported by the Chinese National Foundation of National Sciences (grant no. 30700807), and the Science and Technology project of Liaoning Province (grant no. 2010225032).

\section{References}

1. Jones DH, Nakashima T, Sanchez OH, et al: Regulation of cancer cell migration and bone metastasis by RANKL. Nature 440: 692-696, 2006.

2. Chen L-M, Kuo C-H, Lai T-Y, et al: RANKL increases migration of human lung cancer cells through intercellular adhesion molecule-1 upregulation. J Cell Biochem 112: 933-941, 2011.

3. Sabbota AL, Kim HRC, Zhe X, et al: Shedding of RANKL by tumor-associated MT1-MMP activates Src-dependent prostate cancer cell migration. Cancer Res 70: 5558-5566, 2010.

4. Frezza M, Schmitt S and Dou QP: Targeting the ubiquitin-proteasome pathway: an emerging concept in cancer therapy. Curr Top Med Chem: Aug. 9, 2011 (E-pub ahead of print).

5. Codony-Servat J: Differential cellular and molecular effects of bortezomib, a proteasome inhibitor, in human breast cancer cells. Mol Cancer Ther 5: 665-675, 2006.

6. Teicher BA, Ara G, Herbst R, et al: The proteasome inhibitor PS-341 in cancer therapy. Clin Cancer Res 5: 2638-2645, 1999.

7. Gan Y, Shi C, Inge L, et al: Differential roles of ERK and Akt pathways in regulation of EGFR-mediated signaling and motility in prostate cancer cells. Oncogene 29: 4947-4958, 2010.

8. Jiang Q, Zhou C, Bi Z, et al: EGF-induced cell migration is mediated by ERK and PI3K/AKT pathways in cultured human lens epithelial cells. J Ocul Pharmacol Ther 22: 93-102, 2006.

9. Hwang YP, Yun HJ, Choi JH, et al: Suppression of EGF-induced tumor cell migration and matrix metalloproteinase-9 expression by capsaicin via the inhibition of EGFR-mediated FAK/Akt, PKC/Raf/ERK, p38 MAPK, and AP-1 signaling. Mol Nutr Food Res 55: 594-605, 2011.

10. Armstrong AP, Miller RE, Jones JC, et al: RANKL acts directly on RANK-expressing prostate tumor cells and mediates migration and expression of tumor metastasis genes. Prostate 68: 92-104, 2008

11. Darnay BG, Haridas V, Ni J, et al: Characterization of the intracellular domain of receptor activator of NF-kappaB (RANK). Interaction with tumor necrosis factor receptor-associated factors and activation of NF-kappaB and c-Jun N-terminal kinase. J Biol Chem 273: 20551-20555, 1998.

12. Chen $T$ and Feng X: Cell-based assay strategy for identification of motif-specific RANK signaling pathway inhibitors. Assay Drug Dev Technol 4: 473-482, 2006.

13. Darnay BG, Ni J, Moore PA, et al: Activation of NF-kappaB by RANK requires tumor necrosis factor receptor-associated factor (TRAF) 6 and NF-kappaB-inducing kinase. Identification of a novel TRAF6 interaction motif. J Biol Chem 274: 7724-7731, 1999.

14. Lee SY, Reichlin A, Santana A, et al: TRAF2 is essential for JNK but not NF-kappaB activation and regulates lymphocyte proliferation and survival. Immunity 7: 703-713, 1997.

15. Santini D, Perrone G, Roato I, et al: Expression pattern of receptor activator of NFKB (RANK) in a series of primary solid tumors and related bone metastases. J Cell Physiol 226: 780-784, 2011.

16. Tang ZN, Zhang F, Tang P, et al: RANKL-induced migration of MDA-MB-231 human breast cancer cells via Src and MAPK activation. Oncol Rep 26: 1243-1250, 2011. 
17. Zwickl P, Voges D and Baumeister W: The proteasome: a macromolecular assembly designed for controlled proteolysis. Philos Trans R Soc Lond B Biol Sci 354: 1501-1511, 1999.

18. Lee Y, Hyung SW, Jung HJ, et al: The ubiquitin-mediated degradation of Jak1 modulates osteoclastogenesis by limiting interferon-induced inhibitory signaling. Blood 111: 885-893, 2008.

19. Kim JH, Kim K, Jin HM, et al: Negative feedback control of osteoclast formation through ubiquitin-mediated down-regulation of NFATc1. J Biol Chem 285: 5224-5231, 2009.

20. Adams J: The proteasome: a suitable antineoplastic target. Nat Rev Cancer 4: 349-360, 2004.

21. Adams J, Palombella VJ, Sausville EA, et al: Proteasome inhibitors: a novel class of potent and effective antitumor agents. Cancer Res 59: 2615-2622, 1999.

22. Cusack JC Jr, Liu R, Houston M, et al: Enhanced chemosensitivity to CPT-11 with proteasome inhibitor PS-341: implications for systemic nuclear factor-kappaB inhibition. Cancer Res 61: 3535-3540, 2001.

23. Lorch JH, Thomas TO and Schmoll HJ: Bortezomib inhibits cell-cell adhesion and cell migration and enhances epidermal growth factor receptor inhibitor-induced cell death in squamous cell cancer. Cancer Res 67: 727-734, 2007.
24. Yang CH: Bortezomib (Velcade ${ }^{\circledR}$ ) in metastatic breast cancer: pharmacodynamics, biological effects, and prediction of clinical benefits. Ann Oncol 17: 813-817, 2006.

25. Schmid P, Kuhnhardt D, Kiewe P, et al: A phase I/II study of bortezomib and capecitabine in patients with metastatic breast cancer previously treated with taxanes and/or anthracyclines. Ann Oncol 19: 871-876, 2008.

26. Awada A, Albanell J, Canney PA, et al: Bortezomib/docetaxel combination therapy in patients with anthracycline-pretreated advanced/metastatic breast cancer: a phase I/II dose-escalation study. Br J Cancer 98: 1500-1507, 2008

27. Santini D, Schiavon G, Vincenzi B, et al: Receptor activator of $\mathrm{NF}-\kappa \mathrm{B}$ (RANK) expression in primary tumors associates with bone metastasis occurrence in breast cancer patients. PLoS One 6: e19234, 2011 